\title{
Association of Age and Size of Carotid Artery Intraplaque Hemorrhage and Minor Fibrous Cap Disruption: A High Resolution Magnetic Resonance Imaging Study
}

\author{
Yuanyuan Cui ${ }^{1}$, Huiyu Qiao ${ }^{2}$, Lu Ma ${ }^{1}$, Mingming Lu ${ }^{1}$, Jiafei Yang ${ }^{3}$, Guoen Yao ${ }^{4}$, Jianming Cai ${ }^{1,3}$ and \\ Xihai Zhao ${ }^{2}$ \\ Jianming Cai and Xihai Zhao are joint senior authors. \\ ${ }^{1}$ Department of Radiology, PLA General Hospital, Beijing, China \\ ${ }^{2}$ Center for Biomedical Imaging Research, Department of Biomedical Engineering, Tsinghua University School of Medicine, Beijing, \\ China \\ ${ }^{3}$ Department of Radiology, First Affiliated Hospital of PLA General Hospital, Beijing, China \\ ${ }^{4}$ Department of Neurology, First Affiliated Hospital of PLA General Hospital, Beijing, China
}

Aim: To investigate the association between the volumes of different aging intraplaque hemorrhage (IPH) and minor fibrous cap disruption (MFCD) in carotid arteries.

Methods: Patients with cerebrovascular symptoms and carotid atherosclerotic plaques determined by ultrasound were recruited and underwent multi-contrast magnetic resonance (MR) vessel wall imaging for carotid arteries. Carotid plaques with IPH on MR imaging were included in the analysis. The age (fresh or recent) and the volume of IPH for each plaque were evaluated.

Results: In total, 41 carotid plaques in 37 patients (mean age $70.2 \pm 11.0$ years old; 32 males) were eligible for statistical analysis. The absolute volume of fresh IPH in plaques with MFCD was significantly larger than that in plaques without MFCD $\left(109.83 \pm 75.49 \mathrm{~mm}^{3}\right.$ vs. $\left.30.54 \pm 20.62 \mathrm{~mm}^{3}, P=0.002\right)$. Logistic regression showed that the absolute volume of fresh IPH was significantly associated with MFCD before (odds ratio [OR], 1.735; 95\% confidence interval $[\mathrm{CI}], 1.127-2.670 ; P=0.012)$ and after adjusting for confounding factors $(\mathrm{OR}, 1.823$; 95\% CI, 1.076-3.090; $P=0.026)$. There was no significant association between recent IPH volume and MFCD $(P>0.05)$.

Conclusion: The volume of fresh IPH is independently associated with MFCD in carotid plaques, suggesting that integrity of fibrous cap may change with different age and size of IPH.

Key words: Magnetic resonance imaging, Carotid artery, Atherosclerosis, Stenosis, Cardiovascular diseases

\section{Introduction}

Fibrous cap rupture (FCR) has been demonstrated to be significantly related to future ischemic cerebrovascular events ${ }^{1)}$. Investigators have found that intraplaque hemorrhage (IPH) may stimulate the progression and subsequent FCR of atherosclerotic plaque ${ }^{2,3)}$. Histologically, FCR includes ulceration and minor fibrous cap disruption (MFCD). van Dijk et al. rep- orted that, compared with carotid arteries without IPH, the disrupted plaque surface is more prevalent in those with $\mathrm{IPH}^{3)}$. It is well established that the IPH can be classified into three ages: fresh IPH ( $<1$ week), the major ingredients including intact red blood cell (RBC), lymphocytes, polymorphonuclear cells, and scattered macrophages; recent IPH (1-6 weeks) that is composed of lytic RBC, macrophage cluster, occasional giant cells, cholesterol crystals, and peripheral angiogenesis;

Address for correspondence: Jianming Cai, Department of radiology, PLA General Hospital, 28th Fuxing road, Haidian District, 100853, Beijing, China 
and old IPH ( $>6$ weeks) consisting of amorphous debris and hemosiderin ${ }^{4)}$. The changes of the metabolites of hemoglobin and inflammatory components in different ages of IPH may affect the occurrence of MFCD. Furthermore, the increasing size of IPH, which means more metabolites of hemoglobin and inflammatory components deposition, may also play a role in the occurrence of MFCD. However, the association between the age and size of IPH and the presence of carotid artery MFCD is less evidenced.

Multi-contrast magnetic resonance (MR) vessel wall imaging was demonstrated to have the capability of accurately assessing the morphology and composition of carotid atherosclerosis plaque ${ }^{5)}$. Chu et al. claimed that different ages of IPH are distinguishable on the multi-contrast carotid artery MR images according to their signal intensity patterns on $\mathrm{T} 2$-weighted imaging ${ }^{4)}$. In addition, previous studies have shown that ulceration ${ }^{6}$ and $\mathrm{MFCD}^{7)}$ can be accurately identified by multi-contrast MR vessel wall imaging as well.

\section{Aim}

This study sought to determine the associations between the age and size of IPH and the presence of MFCD in carotid arteries using multi-contrast MR vessel wall imaging techniques.

\section{Methods}

\section{Study Population}

Patients with recent ( $<2$ weeks) ischemic stroke or transient ischemia attack (TIA) and atherosclerotic plaques in at least one carotid artery determined by ultrasound were recruited and underwent MR vessel wall imaging for bilateral carotid arteries in PLA General Hospital. The carotid plaque was diagnosed by ultrasound when the intima-media thickness is equal to or greater than $1.5 \mathrm{~mm}$. Subjects with IPH in carotid arteries were included in the final statistical analysis. Clinical information including age, gender, history of hypertension, smoking, hyperlipidemia, diabetes, and coronary heart disease was collected from clinical record. The study protocol was approved by the institutional review board, and the written consent form was obtained from each subject.

\section{Carotid Artery MR Imaging}

Carotid artery vessel wall imaging was performed on a 3.0-T MR scanner (Signa HDx, General Electric Medical System, Milwaukee, WI, USA) with a 4-channel dedicated phase-arrayed carotid coil. A multi-contrast MR imaging protocol was utilized to acquire threedimensional time-of-flight (3D TOF) and two-dimen- sional T1-weighted (T1W) and T2-weighted (T2W) images with the following parameters: 3D TOF: threedimensional gradient echo, TR/TE 29/2.1 ms, field of view (FOV) $140 \times 140 \mathrm{~mm}^{2}$, matrix size $256 \times 256$, slice thickness $2 \mathrm{~mm}$, flip angle $20^{\circ}$; T1W: fast spin echo (FSE), TR/TE $800 / 8.9 \mathrm{~ms}$, FOV $140 \times 140 \mathrm{~mm}^{2}$, matrix size $256 \times 256$, slice thickness $2 \mathrm{~mm}$, flip angle $90^{\circ}$; and T2W: FSE, TR/TE 3000/57 ms, FOV $140 \times$ $140 \mathrm{~mm}^{2}$, matrix size $256 \times 256$, slice thickness $2 \mathrm{~mm}$, flip angle $90^{\circ}$. The MR scan was centered to the bifurcation of carotid arteries associated with cerebrovascular symptoms. The longitudinal coverage was $24 \mathrm{~mm}$ (12 slices).

\section{MR Image Analysis}

Carotid arteries with IPH were interpreted by two radiologists (C.Y. and Z.X.) who have more than 2 years of experience in cerebrovascular imaging using custom-designed software CASCADE (Vascular Imaging Lab, University of Washington) with consensus. Both radiologists were blinded to clinical information. The image quality of each slice was rated on a 5-point scale dependent on the overall signal-to-noise ratio: $(1=\text { poor, } 5=\text { excellent })^{8)}$. Carotid MR images with poor image quality were excluded. The lumen and outer wall boundaries were outlined at each axial location. The presence or absence and the size of plaque compositions, such as calcification, lipid-rich necrotic core (LRNC), and IPH were identified and measured according to the published criteria ${ }^{9}$. The presence of IPH was defined as hyperintense on T1W and 3D TOF images compared with the adjacent sternocleidomastoid. The IPH was categorized into two types: fresh IPH: intact RBC with intracellular methemoglobin, which showed hyperintense on T1W/3D TOF images and hypointense or isointense on T2W images; and recent IPH: lytic RBC with extracellular methemoglobin, which showed hyperintense on T1W/3D TOF images and hyperintense on T2W images ${ }^{4)}$. The presence or absence of MFCD was determined. The presence of MFCD was defined when the hyperintense of IPH extending into the lumen and the hypointense of a band between the high signal of lumen and juxtaluminal hemorrhage was absent on TOF images ${ }^{5,7)}$. Fig. 1 represents examples of MFCD on MR imaging. The morphology of carotid arteries including lumen area, wall area, total vessel area, maximum wall thickness, and mean wall thickness were also measured. The North American Symptomatic Carotid Endarterectomy Trial criteria ${ }^{10)}$ were utilized to evaluate the degree of carotid artery stenosis on the 3D TOF MRA images after maximum intensity project reconstruction. 

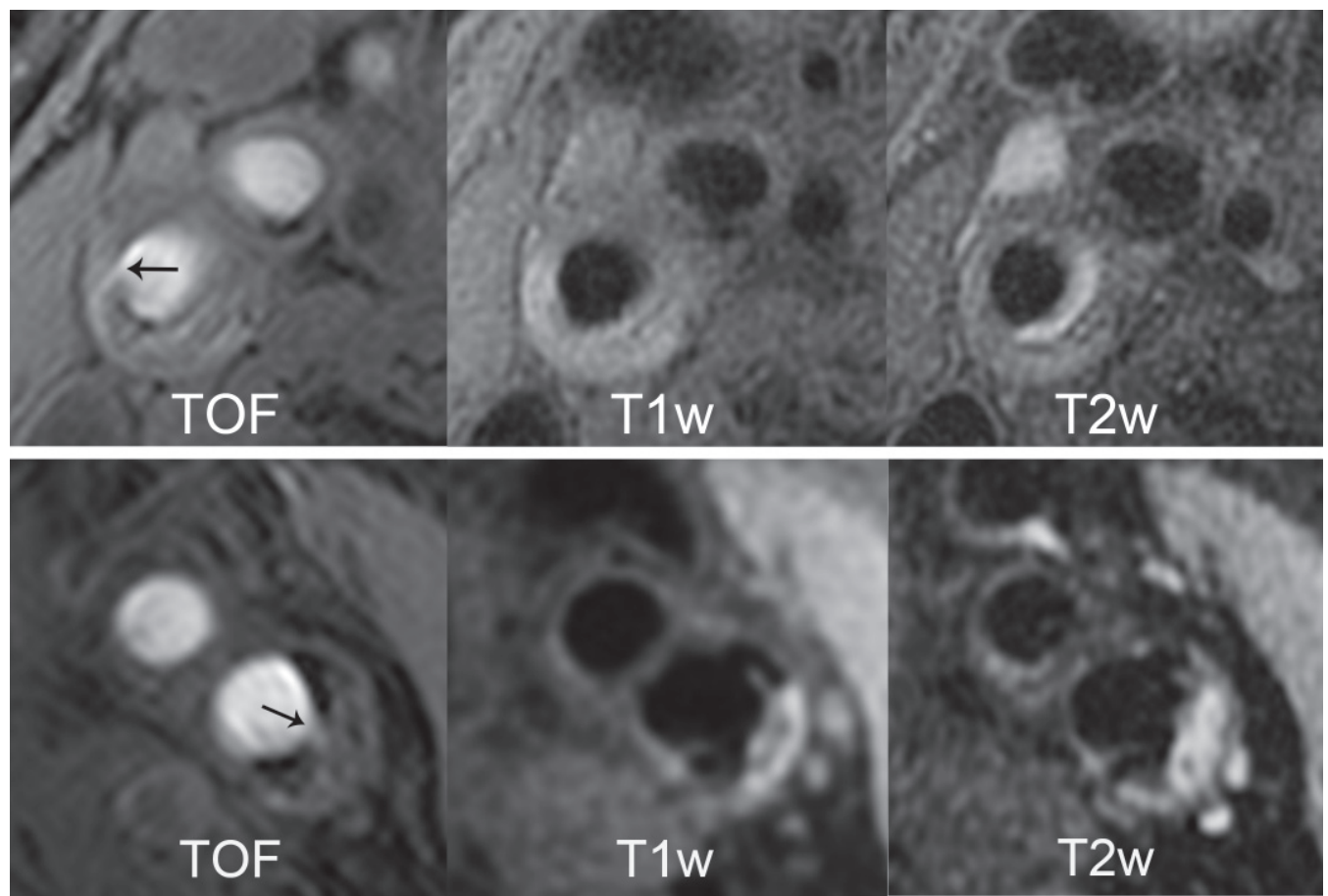

Fig. 1. Illustration of minor fibrous cap disruption (black arrows). The MR images on the upper row and the lower row are from two different cases. On TOF images, juxta-luminal dark band, indicating fibrous cap, was absent and the hyperintense of IPH extended to the lumen.

\section{Statistical Analysis}

Carotid arteries with ulceration, occlusion, or IPH, which was incompletely covered by MR imaging, were excluded from statistical analysis. The percent volume of fresh, recent, and all IPH was defined as the volume of each type of IPH divided by the vessel wall volume in the slices with corresponding type of IPH. The absolute and percent volumes of different types of IPH were compared between carotid plaques having corresponding type of IPH with and without MFCD using Mann-Whitney ranks analysis. Chi-squared test was used to compare the prevalence of MFCD in carotid plaques with fresh and recent IPHs. Spearman's correlation analysis was performed to evaluate the relationships of the absolute and percent volumes of different types of IPH with the presence of MFCD. Univariate regression was used to calculate the odds ratio (OR) and the corresponding $95 \%$ confidence interval (CI) for the absolute and percent volumes of different types of IPH in discriminating the presence of MFCD. Multivariate regression was utilized to determine the associations between the absolute and percent volume of different types of IPH and the presence of MFCD after adjusted for model 1: age and gender; model 2: age, gender, mean wall thickness, and luminal stenosis. C-statistic was conducted to calculate the probability of the absolute volume of different types of IPH in predicting MFCD before and after adjusted for confounding factors, including age, gender, stenosis, and mean wall thickness. Receiver operating characteristic (ROC) curve and area under the curve (AUC) were analyzed to assess the strength of the absolute volume of different types of IPH in discriminating the presence of MFCD. A $P$ value of $<0.05$ was considered as statistically significant. The software of SPSS 22.0 (IBM, Chicago, IL, USA) was used to conduct all statistical analysis.

\section{Results}

In total, 56 patients with carotid IPH determined by MR imaging were recruited from December 2009 to December 2012. Of 112 carotid arteries in 56 recruited patients, 71 arteries were excluded because of the absence of IPH $(n=32)$, having occlusion $(n=9)$, ulceration $(n=20)$, insufficient longitudinal coverage for IPH characterization $(n=9)$, and poor imaging quality $(n=1)$. Finally, 41 carotid arteries in 37 patients (mean age $70.2 \pm 11.0$ years old; 32 males) were eligible for statistics analysis. Of 37 eligible patients, 31 $(83.78 \%)$ had ischemic stroke and $6(16.22 \%)$ had TIA. The clinical characteristics of this study population are summarized in Table 1. 
Table 1. Clinical characteristics of study population $(n=41)$.

\begin{tabular}{lccc}
\hline & \multicolumn{2}{c}{ Mean \pm SD or $\%$} & \\
\cline { 2 - 3 } & $\begin{array}{c}\text { Plaques with MFCD } \\
(n=27)\end{array}$ & $\begin{array}{c}\text { Plaques without MFCD } \\
(n=14)\end{array}$ & \\
\hline Gender, male & $85.7 \%$ & $88.9 \%$ & 0.768 \\
Age, years old & $70.11 \pm 10.67$ & $70.57 \pm 9.59$ & 0.826 \\
Height, $\mathrm{cm}$ & $169.26 \pm 6.33$ & $168.17 \pm 6.49$ & 0.701 \\
Weight, Kg & $69.05 \pm 8.50$ & $70.91 \pm 14.81$ & 0.489 \\
Body mass index, $\mathrm{Kg} / \mathrm{m}^{2}$ & $24.11 \pm 2.47$ & $25.10 \pm 4.58$ & 0.343 \\
Smoking & $17.4 \%$ & $23.1 \%$ & 0.686 \\
Hypertension & $74.1 \%$ & $64.3 \%$ & 0.245 \\
Diabetes & $33.3 \%$ & $21.4 \%$ & 0.476 \\
Coronary heart disease & $29.6 \%$ & $28.6 \%$ & 0.757 \\
Hyperlipidemia & $37.0 \%$ & $28.6 \%$ & 0.782 \\
Low density protein, $\mathrm{mmol} / \mathrm{L}$ & $2.22 \pm 1.51$ & $2.63 \pm 0.89$ & 0.133 \\
High density protein, $\mathrm{mmol} / \mathrm{L}$ & $1.06 \pm 0.51$ & $1.15 \pm 0.28$ & 0.120 \\
Total cholesterol, $\mathrm{mmol} / \mathrm{L}$ & $4.19 \pm 1.91$ & $4.35 \pm 1.03$ & 0.451 \\
Triglyceride, mmol/L & $2.06 \pm 1.52$ & $1.21 \pm 0.35$ & 0.200 \\
\hline
\end{tabular}

MFCD: minor fibrous cap disruption.

Table 2. MR imaging characteristics of carotid atherosclerotic plaques $(n=41)$.

\begin{tabular}{|c|c|c|c|}
\hline & \multicolumn{2}{|c|}{ Mean \pm SD } & \multirow[b]{2}{*}{$P$ value } \\
\hline & $\begin{array}{c}\text { Plaques with MFCD } \\
\qquad(n=27)\end{array}$ & $\begin{array}{l}\text { Plaques without MFCD } \\
\qquad(n=14)\end{array}$ & \\
\hline Maximum wall thickness, $\mathrm{mm}$ & $5.11 \pm 1.82$ & $4.45 \pm 1.52$ & 0.169 \\
\hline Mean wall thickness, mm & $1.72 \pm 0.32$ & $1.47 \pm 0.40$ & 0.014 \\
\hline Stenosis, \% & $35.82 \pm 16.46$ & $28.41 \pm 26.93$ & 0.153 \\
\hline \multicolumn{4}{|l|}{ Absolute volume } \\
\hline LRNC, $\mathrm{mm}^{3}$ & $345.02 \pm 245.69$ & $246.91 \pm 159.94$ & 0.237 \\
\hline Calcification, $\mathrm{mm}^{3}$ & $30.59 \pm 35.33$ & $39.41 \pm 58.52$ & 0.621 \\
\hline${ }^{*}$ Fresh IPH, mm³ & $109.83 \pm 75.49$ & $30.54 \pm 20.62$ & 0.002 \\
\hline${ }^{*}$ Recent IPH, $\mathrm{mm}^{3}$ & $76.57 \pm 98.18$ & $39.02 \pm 28.48$ & 0.920 \\
\hline${ }^{*}$ All IPH, $\mathrm{mm}^{3}$ & $148.30 \pm 94.09$ & $41.69 \pm 37.68$ & $<0.001$ \\
\hline \multicolumn{4}{|l|}{ Percent volume } \\
\hline *Fresh IPH, \% & $21.36 \pm 2.20$ & $10.23 \pm 2.20$ & 0.004 \\
\hline${ }^{*}$ Recent IPH, \% & $12.41 \pm 2.92$ & $8.94 \pm 3.61$ & 0.841 \\
\hline${ }^{*}$ All IPH, \% & $27.46 \pm 8.72$ & $12.78 \pm 10.06$ & $<0.001$ \\
\hline
\end{tabular}

IPH: intraplaque hemorrhage; LRNC: lipid-rich necrotic core; MFCD: minor fibrous cap disruption. * The absolute and percent volumes of each type of IPH were calculated for plaques with corresponding type of IPH.

Imaging Characteristics of Atherosclerotic Plaque

For the 41 carotid plaques with IPH, the maximum and mean wall thickness and luminal stenosis were $4.8 \pm 1.6 \mathrm{~mm}, 1.6 \pm 0.3 \mathrm{~mm}$, and $35.2 \% \pm 20.1 \%$, respectively. Of 41 plaques with IPH, $40(98 \%)$ had fresh IPH, 17 (41\%) had recent IPH, 16 (39.0\%) had both fresh and recent IPH, and 27 (66\%) had MFCD. The mean volume for calcification, LRNC, fresh IPH, recent IPH, and all IPH of all plaques was $36.25 \pm$ $44.73,311.52 \pm 223.08,80.08 \pm 72.73,31.82 \pm 68.90$, and $111.90 \pm 94.00 \mathrm{~mm}^{3}$, respectively.

\section{The Association between Different Types of IPH and MFCD}

There were no significant differences in the prevalence of MFCD between plaques with fresh IPH and 
Table 3. Associations between the size of different type of IPH and MFCD.

\begin{tabular}{|c|c|c|c|c|c|c|}
\hline & \multicolumn{6}{|c|}{ Presence of minor fibrous cap disruption } \\
\hline & \multicolumn{2}{|c|}{ Univariate regression } & \multicolumn{2}{|l|}{ Model 1} & \multicolumn{2}{|l|}{ Model 2} \\
\hline & OR $(95 \% \mathrm{CI})$ & $P$ & OR $(95 \% \mathrm{CI})$ & $P$ & OR $(95 \% \mathrm{CI})$ & $P$ \\
\hline Absolute volume of fresh IPH & $1.735(1.127-2.670)$ & 0.012 & $1.701(1.117-2.590)$ & 0.013 & $1.823(1.076-3.090)$ & 0.026 \\
\hline Absolute volume of recent IPH & $1.355(0.844-2.208)$ & 0.208 & $1.376(0.847-2.237)$ & 0.198 & $1.252(0.771-2.032)$ & 0.363 \\
\hline Absolute volume of all IPH & $2.139(1.269-3.603)$ & 0.004 & $2.164(1.277-3.665)$ & 0.004 & $2.152(1.241-3.730)$ & 0.006 \\
\hline Percent volume of fresh IPH & $1.600(1.115-2.295)$ & 0.011 & $1.607(1.110-2.326)$ & 0.012 & $2.220(1.216-4.054)$ & 0.009 \\
\hline Percent volume of recent IPH & $1.428(0.852-2.394)$ & 0.176 & $1.450(0.858-2.449)$ & 0.165 & $1.296(0.750-2.239)$ & 0.354 \\
\hline Percent volume of all IPH & $2.230(1.377-3.610)$ & 0.001 & $2.233(1.376-3.625)$ & 0.001 & $2.867(1.505-5.461)$ & 0.001 \\
\hline
\end{tabular}

IPH: intraplaque hemorrhage. The OR of absolute volume and percent volume of IPH was calculated by using logistic regression models with increment of $20 \mathrm{~mm}^{3}$ and 5 percent, respectively. Model 1 was adjusted for age and sex; Model 2 was adjusted for age, sex, mean wall thickness and stenosis.

those with recent IPH (65\% vs. $79 \%, P=0.277)$. Compared with plaques without MFCD, those with MFCD had significantly larger mean wall thickness, absolute volume, and percent volume of fresh IPH and all IPH (all $P<0.05$, Table 2). No significant differences were found in the maximum wall thickness, stenosis, absolute volume of LRNC, calcification, and recent IPH and percent volume of recent IPH between plaques with and without MFCD (all $P>0.05$, Table 2). Spearman's correlation analysis revealed that the MFCD was significantly correlated with the absolute volume of fresh IPH $(r=0.483, P=0.001)$, absolute volume of all $\mathrm{IPH}(r=0.669, P<0.001)$, percent volume of fresh IPH $(r=0.463, P=0.003)$, and percent volume of all IPH $(r=0.586, P<0.001)$. There was no significant correlation between the absolute volume $(r=0.246, P=0.121)$ and percent volume of recent IPH $(r=0.229, P=0.156)$ and MFCD.

The results of logistic regression analysis are detailed in Table 3. Univariate logistic regression showed that the OR of the absolute volume and percent volume of fresh IPH was 1.735 (95\% CI, 1.127-2.670, $P=0.012)$ and 1.600 (95\% CI, $1.115-2.295, P=0.011)$ in discriminating the presence of MFCD, respectively. Multivariate logistic regression showed that, in discriminating the presence of MFCD, the OR of the absolute volume and percent volume of fresh IPH was 1.701 (95\% CI, 1.117-2.590, $P=0.013)$ and 1.607 (95\% CI, $1.110-2.326, P=0.012)$ after adjusting for age and sex (model 1) and 1.823 (95\% CI, 1.076-3.090, $P=$ $0.026)$ and $2.220(95 \%$ CI, 1.216-4.054, $P=0.009)$ after adjusting for age, sex, stenosis, and mean wall thickness (model 2), respectively. In contrast, there were no significant associations between the absolute volume and percent volume of recent IPH and the presence of MFCD before and after adjusting for confounding factors (all $P>0.05$ ).
In discriminating the presence of MFCD, ROC curve analysis showed that the absolute volume for all $\mathrm{IPH}$ had the highest AUC (AUC $=0.907,95 \%$ CI $0.805-1.000)$, followed by fresh IPH $(\mathrm{AUC}=0.794$, $95 \%$ CI $0.658-0.930)$, and recent IPH $(\mathrm{AUC}=0.638$, 95\% CI 0.462-0.813) (Fig. 2). After adjusted for confounding factors including age, gender, stenosis, and mean wall thickness, the AUC for absolute volume of all, fresh, and recent IPH was 0.905 (CI\% $0.802-$ 1.000), 0.822 (CI\% 0.692-0.953), and 0.728 (CI\% $0.555-0.901$ ), respectively (Fig.2). Fig. 3 is an example showing the occurrence of MFCD in a carotid plaque with fresh IPH.

\section{Discussion}

This study investigated the relationship between the age and size of IPH and MFCD in carotid arteries. We found that the volume of fresh IPH in carotid plaques with MFCD was significantly larger than that in plaques without MFCD. Significant correlations can be found between the absolute volume and percent volume of fresh IPH and the presence of MFCD before and after adjusted for confounding factors. However, significant associations were not observed between the size of recent IPH and the presence of MFCD. Our findings indicate that the size of fresh IPH might be an independent indicator for MFCD in carotid atherosclerotic plaques.

In the present study, the volume of fresh IPH was found to be significantly associated with the presence of MFCD. Previous study has shown that IPH was a strong predictor for FCR ${ }^{11)}$ in carotid atherosclerotic plaques. However, in the above study, the role of IPH in different age and size in predicting FCR has not been investigated. Investigators have reported that the presence of IPH will stimulate the progression of 
plaque $^{12,13)}$. However, to the best of our knowledge, there is no reported evidence on the association between the size of fresh IPH and plaque progression. An MR imaging and histology comparison study showed that the major ingredients of fresh IPH include intact RBC, lymphocytes, polymorphonuclear cells, and scattered macrophages ${ }^{4)}$. The inflammatory activity within atherosclerotic plaque with IPH will lead to the instability of plaque surface ${ }^{14)}$. In the initial phase of IPH, leukocytes, particularly neutrophils, and plasma zymogen were conveyed in plaque with the RBC entrance. These two components of fresh IPH were the key sources of proteases, such as coagulation proteases, leukocyte serine proteases, and gelatinase, which accounted for a major part of the proteolytic degradation of fibrous cap ${ }^{15)}$. Neutrophil gelatinase and serine protease are mainly conveyed by bleeding within plaques, which are capable of degrading the extracellular matrix. Besides, serine proteases were found to play a role in degrading atheroprotective proteins released by smooth muscle cells ${ }^{16)}$. The increases of the size and complexity of the plaque due to IPH will subsequently increase the amount of activated proteases within plaque ${ }^{17)}$. Therefore, with increases of fresh IPH in plaque, the subsequently potent inflammatory action in this plaque will aggravate the disruption of fibrous cap.

The biomechanical propriety of IPH might play a role in the vulnerability of plaque surface ${ }^{18)}$. It has been shown that IPH had a close connection with enhanced stress over fibrous cap ${ }^{19,20)}$. According to previous investigators, because of the different ingredients appearing during aging of IPH, the mechanical impact of IPH on fibrous cap will change dynamically. It is reported that the stress over fibrous cap increases from $118 \mathrm{kPa}$ (79-189) to $159 \mathrm{kPa}(114-253)$ if simulation was done by replacing the lipid pool with fresh IPH. And a 30\% decrease was observed if the fresh IPH was replaced by recent or old IPH. The pathomechanical explanation is that the fresh IPH is inclined to undergo massive deformations that increase the stress over the plaque surface under blood pressure loading ${ }^{21)}$. The biomechanical mechanism behind the relationship between the age of IPH and MFCD needs further investigation.

In the present study, the volume of all IPH had a greater AUC than that of fresh IPH in discriminating the presence of MFCD. This indicates that the recent IPH may also play a role in the rupturing progress of fibrous cap. Histologically, the recent IPH includes lytic $\mathrm{RBC}$ with extracellular methemoglobin and cluster of macrophages. Macrophages within plaque were able to secrete lytic enzymes that may be responsible for the impairing of the fibrous cap and subsequent rupture of the plaque surface ${ }^{22)}$. Therefore, the higher density of macrophages in plaques, especially under the fibrous
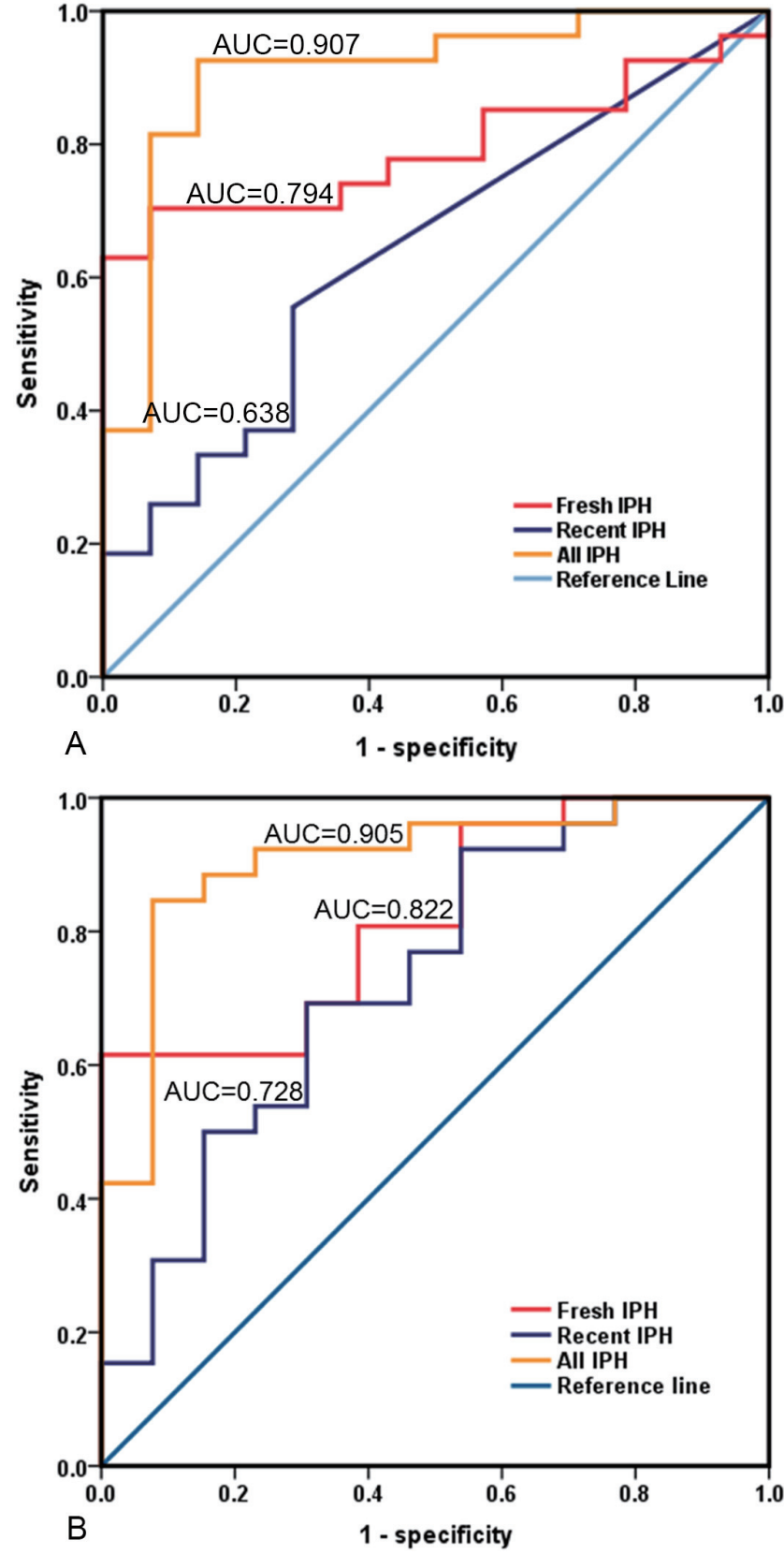

Fig. 2. The ROC curves for the volume of different ages of $\mathrm{IPH}$ in predicting MFCD. Figures A and B represent the AUC values of different types of IPH before and after adjusted for confounding factors, respectively.

AUC: area under the curve; Fresh IPH: the volume of fresh IPH; Recent IPH: the volume of recent IPH; All IPH: the volume of all IPH.

cap, is attributed to the plaques' rupture ${ }^{23,24)}$. In addition, the hemoglobin released from the RBCs in the recent IPH plays a part in destabilizing the plaque because of its potent pro-inflammatory nature, thereby promoting the formation of reactive oxygen species $^{25)}$. 


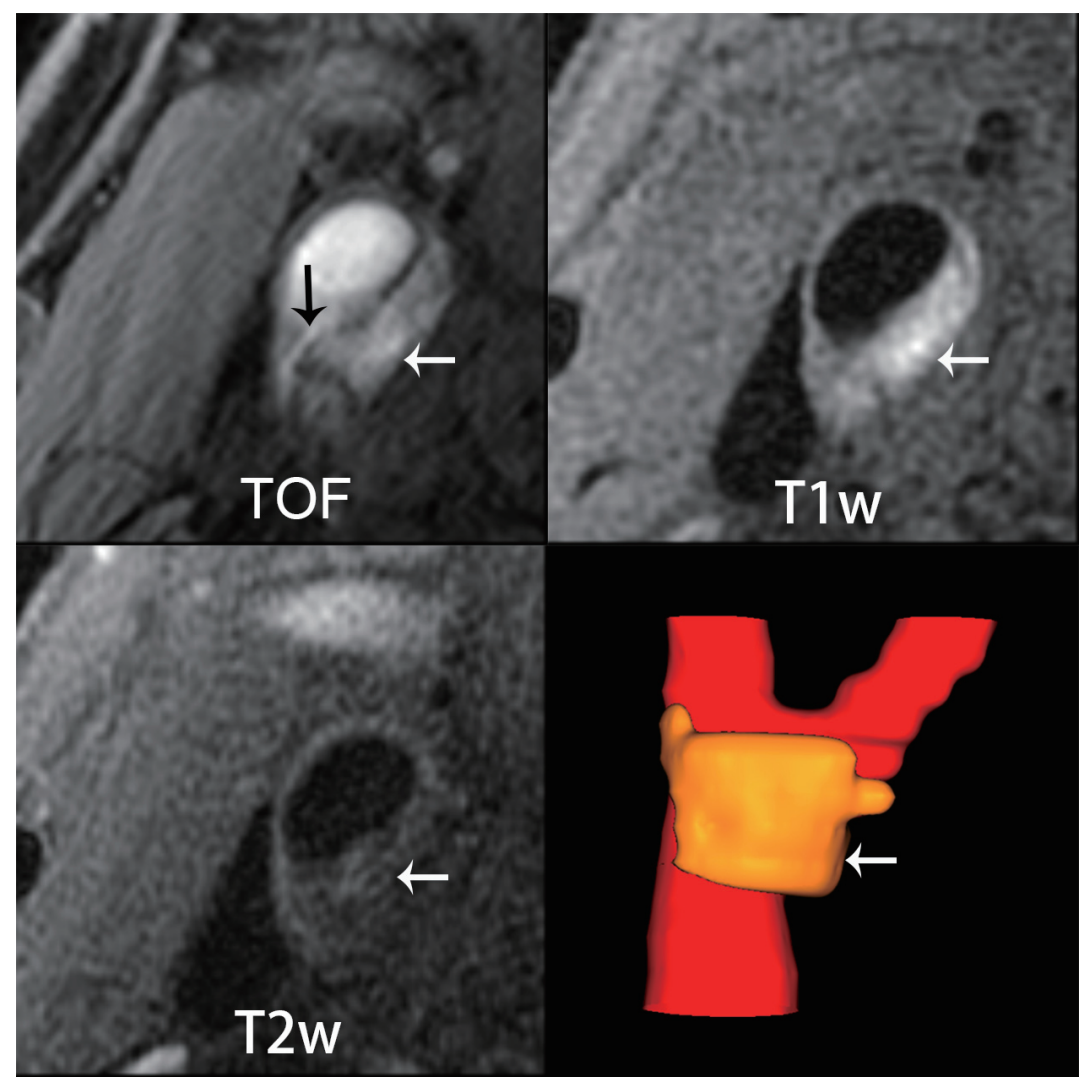

Fig. 3. The MR images from a 62-year-old male patient showed eccentric plaque with fresh IPH (white arrows) and minor fibrous cap disruption (black arrow) on the right common carotid artery. A large volume of fresh IPH can be seen on volume rendering (VR) image (white arrow).

However, according to the results in the present study, compared with fresh IPH, the roles recent IPH played in destabilizing the plaque are weaker. On the other hand, the increase of volume of all IPH including fresh and recent IPH would augment the size of LRNC. The volume of LRNC was found to be the strongest predictor for FCR ${ }^{26,27)}$. With the increase of IPH volume regardless of its age, the biomechanical environment of plaque surface may alter. Teng et al. demonstrated that the stretch was high around the ruptured plaque site in the plaque with juxta-luminal hemorrhage $^{28)}$. For carotid plaques, the larger volume of IPH to have, the more interface of IPH to conjunct with plaque surface.

In previous studies, most of the investigators believed that MFCD was one of the sequelae of plaque progression stimulated by IPH. However, in a recent histological study ${ }^{29)}$, it was assumed that fissured MFCD might be a potential resource of IPH that was usually underestimated. In the above study, investigators found a track of blood in the fissured area of plaque surface and it expended to the LRNC and the matrix of calci- fications ${ }^{29)}$. Similar phenomenon has been reported in an intravascular ultrasound study showing flowing blood in coronary plaque fissures ${ }^{30)}$. In the above histological study, 63\% of plaques with MFCD had fresh IPH. Therefore, it seems to be controversial whether the MFCD is a sequela or a potential resource of IPH.

Our study has several limitations. First, the sample size of this study was small. Future studies with larger sample size are suggested. Second, a few of the arteries were excluded because of the incomplete coverage of IPH. 3D MR imaging techniques, such as magnetization-prepared rapid acquisition gradient echo (MPRAGE) and simultaneous non-contrast angiography and $\mathrm{IPH}$, could be potentially used to provide a sufficient longitudinal coverage in future studies. Third, in the present study, T1W and TOF imaging sequences were the key techniques to characterize IPH. It has been demonstrated that the MP-RAGE imaging technique exhibited a higher sensitivity and specificity in the identification of IPH compared with T1W and TOF imaging ${ }^{31,32)}$. Fourth, the old IPH and its association with MFCD were not determined in this study. It is because 
that there are difficulties in differentiating hemosiderin from calcification using the current MR vessel wall imaging techniques. Yang et al. utilized susceptibility weighted imaging to evaluate the calcification within plaque in femoral arteries ${ }^{33)}$. This technique may have the potential to distinguish hemosiderin from calcifications. Fifth, the in-plane spatial resolution of this study was $0.55 \mathrm{~mm} \times 0.55 \mathrm{~mm}$, which is limited in the identification of small fibrous cap disruption. High spatial resolution may improve the identification of MFCD. Finally, this is a cross-sectional study that cannot capture the nature of plaques with fresh IPH progressing to disrupted lesions. Prospective studies may provide a clue to it.

\section{Conclusion}

The volume of fresh IPH is independently associated with MFCD in carotid plaques, suggesting that the age and size of IPH may play a role in reducing the stability of fibrous cap. Larger fresh IPH may reflect higher possibility of occurrence of MFCD in atherosclerotic plaque.

\section{Acknowledgements}

None.

\section{Conflicts of Interest}

None.

\section{Notice}

This study is supported by the Beijing Municipal Science and Technology Project (Z161100000516194 and D171100003017003) and the National Natural Science Foundation of China (81771825 and 81271536).

\section{Abbreviations}

MR: magnetic resonance

LRNC: lipid-rich necrotic core

IPH: intraplaque hemorrhage

MFCD: minor fibrous cap disruption

FCR: fibrous cap rupture

OR: odds ratio

CI: confidence interval

ROC curve: receiver operating characteristic curve

AUC: area under the curve

\section{References}

1) Gupta A, Baradaran H, Schweitzer AD, Kamel H, Pandya A, Delgado D, Dunning A, Mushlin AI, Sanelli PC: Carotid plaque MRI and stroke risk: a systematic review and meta-analysis. Stroke, 2013; 44: 3071-3077

2) Treiman GS, McNally JS, Kim SE, Parker DL: Correlation of carotid intraplaque hemorrhage and stroke using 1.5 T and 3 T MRI. Magn Reson Insights, 2015; 8: 1-8

3) van Dijk AC, Truijman MT, Hussain B, Zadi T, Saiedie G, de Rotte AA, Liem MI, van der Steen AF, Daemen MJ, Koudstaal PJ, Nederkoorn PJ, Hendrikse J, Kooi ME, van der Lugt A: Intraplaque hemorrhage and the plaque surface in carotid atherosclerosis: the plaque at risk study (PARISK). AJNR Am J Neuroradiol, 2015; 36: $2127-$ 2133

4) Chu B, Kampschulte A, Ferguson MS, Kerwin WS, Yarnykh VL, O'Brien KD, Polissar NL, Hatsukami TS, Yuan C: Hemorrhage in the atherosclerotic carotid plaque: a high-resolution MRI study. Stroke, 2004; 35: 1079 1084

5) Cai JM, Hatsukami TS, Ferguson MS, Small R, Polissar NL, Chun Y. Classification of human carotid atherosclerotic lesions with in vivo multicontrast magnetic resonance imaging: Circulation, 2002; 106: 1368-1373

6) Yu W, Underhill HR, Ferguson MS, Hippe DS, Hatsukami TS, Yuan C, Chu B: The added value of longitudinal black-blood cardiovascular magnetic resonance angiography in the cross sectional identification of carotid atherosclerotic ulceration. Cardiovasc Magn Reson, 2009; 11: 31

7) Kampschulte A, Ferguson MS, Kerwin WS, Polissar NL, Chu B, Saam T, Hatsukami TS, Yuan C: Differentiation of intraplaque versus juxtaluminal hemorrhage/thrombus in advanced human carotid atherosclerotic lesions by in vivo magnetic resonance imaging. Circulation, 2004; 110: 3239-3244

8) Yuan C, Mitsumori LM, Ferguson MS, Polissar NL, Echelard D, Ortiz G, Small R, Davies JW, Kerwin WS, Hatsukami TS: In vivo accuracy of multispectral magnetic resonance imaging for identifying lipid-rich necrotic cores and intraplaque hemorrhage in advanced human carotid plaques. Circulation, 2001; 104: 2051-2056

9) Saam T, Ferguson MS, Yarnykh VL, Takaya N, Xu D, Polissar NL, Hatsukami TS, Yuan C: Quantitative evaluation of carotid plaque composition by in vivo MRI. Arterioscler Thromb Vasc Biol, 2005; 25: 234-239

10) North American Symptomatic Carotid Endarterectomy Trial Collaborators: Beneficial effect of carotid endarterectomy in symptomatic patients with high-grade carotid stenosis. N Engl J Med, 1991; 325: 445-453

11) Xu D, Hippe DS, Underhill HR, Oikawa-Wakayama M, Dong L, Yamada K, Yuan C, Hatsukami TS: Prediction of high-risk plaque development and plaque progression with the carotid atherosclerosis score. JACC Cardiovasc Imaging, 2014; 7: 366-373

12) Takaya N, Yuan C, Chu B, Saam T, Polissar NL, Jarvik GP, Isaac C, McDonough J, Natiello C, Small R, Ferguson MS, Hatsukami TS: Presence of intraplaque hemorrhage stimulates progression of carotid atherosclerotic plaques: a high-resolution magnetic resonance imaging study. Circu- 
lation, 2005; 111: 2768-2775

13) Sun J, Underhill HR, Hippe DS, Xue Y, Yuan C, Hatsukami TS: Sustained acceleration in carotid atherosclerotic plaque progression with intraplaque hemorrhage: a longterm time course study. JACC Cardiovasc Imaging, 2012; 5: 798-804

14) de Vries MR, Quax PH: Plaque angiogenesis and its relation to inflammation and atherosclerotic plaque destabilization. Curr Opin Lipidol, 2016; 27: 499-506

15) Michel JB, Delbosc S, Ho-Tin-Noé B, Leseche G, Nicoletti A, Meilhac O, Martin-Ventura JL: From intraplaque haemorrhages to plaque vulnerability: biological consequences of intraplaque haemorrhages. J Cardiovasc Med (Hagerstown), 2012; 13: 628-634

16) Michel JB, Virmani R, Arbustini E, Pasterkamp G: Intraplaque haemorrhages as the trigger of plaque vulnerability. Eur Heart J, 2011; 32: 1977-1985, 1985a, 1985b, 1985c

17) Leclercq A, Houard X, Loyau S, Philippe M, Sebbag U, Meilhac O, Michel JB: Topology of protease activities reflects atherothrombotic plaque complexity. Atherosclerosis, 2007; 191: 1-10

18) Teng Z, Sadat U, Brown AJ, Gillard JH: Plaque hemorrhage in carotid artery disease: pathogenesis, clinical and biomechanical considerations. J Biomech, 2014; 47: 847858

19) Sadat U, Teng Z, Young VE, Li ZY, Gillard JH: Utility of magnetic resonance imaging-based finite element analysis for the biomechanical stress analysis of hemorrhagic and non-hemorrhagic carotid plaques. Circ J, 2011; 75: 884889

20) Huang $X$, Teng $Z$, Canton G, Ferguson M, Yuan C, Tang $D$ : Intraplaque hemorrhage is associated with higher structural stresses in human atherosclerotic plaques: an in vivo MRI-based 3d fluid-structure interaction study. Biomed Eng Online, 2010; 9: 86

21) Sadat U, Teng Z, Young VE, Zhu C, Tang TY, Graves MJ, Gillard JH: Impact of plaque haemorrhage and its age on structural stresses in atherosclerotic plaques of patients with carotid artery disease: an MR imaging-based finite element simulation study. Int J Cardiovasc Imaging, 2011; 27: 397-402

22) Welgus HG, Campbell EJ, Cury JD, Eisen AZ, Senior RM, Wilhelm SM, Goldberg GI: Neutral metalloproteinases produced by human mononuclear phagocytes. Enzyme profile, regulation, and expression during cellular development. J Clin Invest, 1990; 86: 1496-1502

23) Kodama T, Narula N, Agozzino M, Arbustini E: Pathology of plaque haemorrhage and neovascularization of cor- onary artery. J Cardiovasc Med (Hagerstown), 2012; 13: 620-627

24) Moreno PR, Falk E, Palacious IF, Newell JB, Fuster V, Fallon JT: Macrophages infiltration in acute coronary syndromes. Implications for plaque rupture. Circulation, 1995; 90: 775-778

25) Levy AP, Moreno PR: Intraplaque hemorrhage. Curr Mol Med, 2006; 16: 479-488

26) Underhill HR, Yuan C, Yarnykh VL, Chu B, Oikawa M, Dong L: Predictors of surface disruption with MR imaging in asymptomatic carotid artery stenosis. AJNR Am J Neuroradiol, 1995; 31: 487-493

27) Shah PK: Mechanisms of plaque vulnerability and rupture. J Am Coll Cardiol, 2003; 41: S15-S22

28) Teng Z, Sadat U, Huang Y, Young VE, Graves MJ, Lu J, Gillard JH: In vivo mri-based 3D mechanical stress-strain profiles of carotid plaques with juxtaluminal plaque haemorrhage: an exploratory study for the mechanism of subsequent cerebrovascular events. Eur J Vasc Endovasc Surg, 2011; 42: 427-433

29) Daemen MJ, Ferguson MS, Gijsen FJ, Hippe DS, Kooi ME, Demarco K, van der Wal AC, Yuan C, Hatsukami TS: Carotid plaque fissure: an underestimated source of intraplaque hemorrhage. Atherosclerosis, 2016; 254: 102108

30) Maehara A, Mintz GS, Bui AB, Castagna MT, Walter OR, Pappas C, Pinnow EE, Pichard AD, Satler LF, Waksman R, Suddath WO, Laird JR Jr, Kent KM, Weissman NJ: Incidence, morphology, angiographic findings, and outcomes of intramural hematomas after percutaneous coronary interventions: an intravascular ultrasound study. Circulation, 2002; 105: 2037-2042

31) Ota H, Yarnykh VL, Ferguson MS, Underhill HR, Demarco JK, Zhu DC, Oikawa M, Dong L, Zhao X, Collar A, Hatsukami TS, Yuan C: Carotid intraplaque hemorrhage imaging at 3.0-T MR imaging: comparison of the diagnostic performance of three t1-weighted sequences. Radiology, 2010; 254: 551-563

32) Wang J, Bornert P, Zhao H, Hippe DS, Zhao X, Balu N, Ferguson MS, Hatsukami TS, Xu J, Yuan C, Kerwin WS: Simultaneous noncontrast angiography and intraplaque hemorrhage (SNAP) imaging for carotid atherosclerotic disease evaluation. Magn Reson Med, 2013; 69: 337-345

33) Yang Q, Liu J, Barnes SR, Wu Z, Li K, Neelavalli J, Hu J, Haacke EM: Imaging the vessel wall in major peripheral arteries using susceptibility-weighted imaging. J Magn Reson Imaging, 2009; 30: 357-365 